\title{
O Conceito de Processo de Trabalho para Alinhar Sistemas de Informação com os Objetivos das Organizações
}

\author{
Amauri Marques da Cunha ${ }^{1}$, Gilberto Quirgo de Souza ${ }^{2}$ \\ ${ }^{1}$ Núcleo de Computação Eletrônica - Universidade Federal do Rio de Janeiro (UFRJ) \\ Caixa Postal 2324 - 20.001-970 - Rio de Janeiro - RJ - Brasil \\ ${ }^{2}$ Curso de Graduação em Computação - Fund. Educacional Serra dos Órgãos (FESO) \\ Caixa Postal 93401 - CEP 25965-000 - Teresópolis - RJ - Brasil \\ amauri@nce.ufrj.br, gilbertoqsouza@yahoo.com.br
}

\begin{abstract}
This article introduces the concept of Work Process that can be used by IT professionals in analyzing and making improvements within organizations context. This concept can provide a rigorous approach, which enables the generation of process models that can be traceable with the corresponding information systems models. This is essential to ensure the desired alignment of IT solutions with business objectives.
\end{abstract}

Resumo. Este artigo apresenta o conceito de Processo de Trabalho que pode ser usado por profissionais de TI para analisar e fazer melhorias no contexto das organizações. Este conceito pode prover uma abordagem rigorosa, a qual permite a criação de modelos de processo que podem ser rastreáveis com os correspondentes modelos de sistemas de informação. Isto é essencial para assegurar o alinhamento de soluções de TI com os objetivos do negócio.

\section{Introdução}

O surpreendente desenvolvimento das novas Tecnologias da Informação (TI), que tem sido observado durante os últimos anos, aumentou a quantidade e a variedade dos dispositivos que encapsulam tais tecnologias. As pessoas em geral e os usuários de TI em particular são atingidos constantemente pelos mais diversos anúncios de novos produtos, muitas vezes prometendo maravilhas às organizações que os adotem. Não é possível ignorar tantas novas possibilidades.

Entretanto, o grande desafio continua o mesmo: como estas tecnologias podem ser utilizadas para melhorar a produtividade das organizações como um todo? Para lidar com esta complicada questão, o profissional de TI é induzido freqüentemente a aplicar uma determinada tecnologia ou um dispositivo específico para resolver o problema para o qual tenha sido designado. Em geral, este tipo de procedimento é considerado como "má engenharia". Em uma "boa prática de engenharia", a escolha de uma tecnologia ou de um dispositivo deve ocorrer somente após uma análise completa do problema alvo.

A Engenharia de Software, segundo Pressman (2001), emergiu como uma disciplina que fornece conceitos e ferramentas para a transformação da atividade do profissional de TI em uma verdadeira atividade de engenharia. Além disso, Pressman observa que a "crise do software", que tem persistido nas últimas décadas apesar do 
impressionante progresso tecnológico, é devida à falta de métodos e técnicas bem fundamentados, contrastando com outras áreas da engenharia.

Neste contexto, é introduzido o conceito de Processo de Trabalho para contribuir na construção de um arcabouço formal que ajude a entender o trabalho realizado pelas organizações, a fim de decidir de maneira adequada, como, onde e quando aplicar dispositivos de TI. Este conceito pode ser utilizado em todos os tipos de organização, fornecendo um modelo de referência para que os profissionais de TI representem os ambientes produtivos de maneira bem objetiva.

Este esforço é feito no sentido de identificar importantes conceitos dominados pelos "profissionais experientes de TI", a fim revelar e organizar seu conhecimento tácito. O conceito de Processo de Trabalho é apenas um, dentre os que devem ser desvendados, na construção de uma apropriada "disciplina de engenharia" para o desenvolvimento de software.

Este artigo está dividido em 6 seções. A seção 2 comenta alguns trabalhos relacionados, enquanto a seção 3 discorre sobre o conceito de processo, com o objetivo de apresentar o conceito de Processo de Trabalho na seção 4. Uma discussão sobre o este conceito no ambiente organizacional é feita na seção 5. A última seção apresenta conclusões e esboça oportunidades de trabalhos futuros.

\section{Trabalhos Relacionados}

O desafio de transformar a área de software em uma atividade de engenharia foi reconhecido como crucial pela OMG (Object Management Group), que propôs a abordagem conhecida como MDA (Model Driven Architecture) (Miller 2003). A idéia principal da MDA é promover uma separação entre os níveis de especificação de sistemas de software e o da tecnologia empregada para construí-los, através do estabelecimento de um modelo para cada nível, que são respectivamente o PIM (Platform Independent Model) e o PSM (Platform Specific Model).

A MDA conceituou a transformação de modelos PIM em modelos PSM, a fim de alcançar a desejada separação de níveis. Kleppe (2003) mostrou como esta idéia poderia ser realizada na prática.

O mesmo autor assinala que os requisitos do sistema são a entrada para a fase de análise, a qual produz os modelos PIM como saída. Na medida em que os requisitos dos sistemas são em sua maioria descritos de maneira textual, mesmo quando são utilizadas ferramentas de gerência de requisitos, pode-se argumentar que esta é uma maneira muito pouco rigorosa de expressão. Este tipo de modelagem de requisitos não favorece o rastreamento entre os modelos, tornando mais difícil alcançar transformações mais automatizadas de modelos, o que é um dos principais objetivos da MDA.

Para lidar com esta questão, a MDA definiu uma camada de modelos de nível mais alto, chamada CIM (Computation Independent Model). Esta camada corresponde ao que é conhecido como modelos de negócio ou modelos de domínio, e pretende descrever a organização como um todo, incluindo os sistemas de informação existentes no seu interior, sejam eles compostos ou não por peças de software.

Nossa proposta de Processo do Trabalho situa-se no nível da camada CIM. Seu objetivo principal é fornecer ao profissional de TI, uma ferramenta conceitual para 
entender e representar o trabalho feito dentro da organização, em um nível adequado de abstração, permitindo que tais modelos sejam utilizados como poderosa forma de comunicação entre profissionais de TI e profissionais de negócio.

Ao aplicar o conceito do Processo do Trabalho, espera-se que requisitos mais corretos possam ser extraídos da fase de análise, uma vez que eles estarão naturalmente alinhados com os objetivos da organização. Esta foi a idéia principal de Jacobson (1994) ao propor o uso da Orientação a Objeto para a construção de modelos dos processos que representem o trabalho completo das organizações. A proposta de Jacobson utiliza a abordagem BPR (Business Process Reengineering), que preconiza que as atividades relevantes executadas pelas organizações podem ser estruturadas em processos. Este ponto de vista pode conduzir a um grande aumento de produtividade, devido ao seu bom alinhamento com a maneira de produzir valor para o cliente do processo (Davenport 1994), que é a razão principal da existência dos processos.

A reflexão de Jacobson foi: se for possível representar um processamento de informação, que ocasionalmente pode ser um elemento do software, dentro de uma especificação formal de um processo de negócio, tal elemento do software seria, conseqüentemente, inteiramente alinhado com os objetivos do negócio.

Desafiadoras perguntas permanecem: como escolher os processos apropriados e, quando são escolhidos, como saber se estão suficientemente identificados e definidos? O conceito do Processo do Trabalho tenta responder à última pergunta.

Por outro lado, Gulledge (2001) enfatiza a inexistência de organizações inteiramente orientadas a processos, como seria a situação ideal para alinhar completamente a TI com os objetivos do negócio. De fato, a "cultura hierárquica tradicional" continua resistente e sólida dentro das organizações.

De acordo com nossa experiência profissional, as organizações que têm a ousadia de representar explicitamente os seus próprios processos, a fim estabilizá-los, ainda mantêm as fronteiras hierárquicas funcionais em seus modelos de processo. Este comportamento parece ser o "estado da arte" na aplicação da abordagem de processos no mundo real, e sinaliza a existência de enormes barreiras a esta mudança cultural.

O conceito do Processo de Trabalho permite o tratamento de todos os tipos de processos do negócio, limitados ou não pelas fronteiras funcionais internas. Além disso, a aplicação do conceito de Processo de Trabalho pode mostrar uma maneira de alcançar uma representação mais rigorosa dos processos, com o objetivo de explicitar requisitos para sistemas de software no nível CIM da MDA. Tais requisitos deverão possuir uma boa rastreabilidade com os modelos PIM encontrados nas propostas da MDA.

\section{O Conceito de Processo nas Organizações}

Para aplicar TI de maneira efetiva, é necessário entender o trabalho realizado (por pessoas) dentro das organizações. Um modo objetivo de fazê-lo é através do uso do conceito de processo (Campos 1992) (Davenport 1994).

Entretanto, no mundo dos negócios é encontrada muita imprecisão no uso deste termo. A palavra "processo" é utilizada nas organizações para designar, tanto coisas muito amplas e complexas por vezes chamadas de "macro-processos", quanto coisas pequenas e simples que poderiam facilmente ser chamadas de "atividade" ou "tarefa". 
Outras vezes este termo é utilizado de forma mais indefinida ainda, em frases do tipo: "a empresa está em processo de adaptação a uma nova conjuntura".

Mesmo na literatura recente dedicada ao estudo dos processos nos negócios, como em (Harmon 2003), há um surpreendente tratamento informal do conceito de processo. Parece existir um acordo tácito de que processo é algo tão simples e evidente, que nem necessita ser rigorosamente definido. Porém, isto se constitui numa importante fonte de incompreensões e mal-entendidos, os quais são causas fundamentais da conhecida dificuldade de concepção de soluções de TI alinhadas com o negócio.

É interessante observar uma inconsistência de Harmon (2003) e de muitos outros autores desta área. Quase todos citam Davenport (1994) como uma referência básica para o conceito de processo, mas não mantêm fidelidade ao conceito. De acordo com Davenport (1994), um processo é um conjunto das atividades que devem ser executadas para atender a um cliente, é uma estrutura específica de atividades localizada no tempo e no espaço, com um começo, um fim, entradas e saídas claramente identificadas.

Usar a abordagem de processos implica em uma visão horizontal do negócio, e numa redução da ênfase na estrutura hierárquica da organização. Significa ainda que as interfaces entre unidades funcionais devem ser melhoradas ou eliminadas, o que provoca um inevitável conflito com a estrutura hierárquica funcional, mas pode proporcionar enormes ganhos de produtividade, especialmente quando acompanhada por uso intensivo de TI (Davenport 1994). Para viabilizar a aplicação destas idéias, é proposta a seguir uma conceituação mais rigorosa de Processo de Trabalho.

\section{O Conceito de Processo de Trabalho}

As chamadas tecnologias da informação (sobretudo Informática e Telecomunicações) são fundamentadas nas ciências exatas, que utilizam o formalismo e a lógica matemática em todos os seus raciocínios. Conseqüentemente, elas precisam de um nível similar de formalização nas áreas onde são aplicadas.

Não é possível, por exemplo, exigir de um dispositivo de TI um comportamento adequado diante de uma situação para a qual ele não foi previsto. Isto causa dificuldades para o mundo dos negócios, que está acostumado a utilizar seres humanos como dispositivos processadores de informação, os quais são capazes de tomar decisões adequadas em situações nunca enfrentadas anteriormente. Esta é uma causa importante de decepções com o uso de TI: a inflação das expectativas de desempenho.

A solução clássica para esta questão é a criação de um arcabouço conceitual que possa ser compartilhado pelos integrantes do mundo dos negócios e pelos especialistas em TI. Nesta linha já existem experiências bem sucedidas, como por exemplo, a modelagem conceitual de dados utilizando o modelo E-R para a comunicação entre os dois tipos de profissionais. É necessário e oportuno criar um modelo conceitual para processos, que seja totalmente independente de qualquer tecnologia específica, ao mesmo tempo em que seja suficientemente rigoroso para permitir análises e decisões sobre o uso de TI no próprio processo.

Processo de Trabalho é conceituado como sendo constituído por um conjunto de atividades que devem ser executadas para produzir pelo menos um resultado identificável e utilizável por um ente denominado cliente do processo de trabalho. $\mathrm{O}$ processo de trabalho deve ter fronteiras claramente identificadas pelas suas entradas e 
saídas. Cada saída é denominada um resultado do processo de trabalho e cada entrada um acionamento do processo de trabalho.

Como o processo de trabalho pode possuir mais de um tipo de resultado, ele também pode possuir mais de um tipo de cliente. Ele pode ter, no máximo, um tipo de cliente para cada tipo de resultado que oferece como saída. Ele deve ter, no mínimo, um tipo de resultado e um tipo de cliente para ser considerado um processo de trabalho. É importante ressaltar que tipo de cliente é um papel desempenhado por uma pessoa, um grupo de pessoas, um outro processo de trabalho, ou até um dispositivo de TI, que seja capaz de identificar e utilizar o tipo de resultado correspondente, que foi enviado como saída pelo processo de trabalho.

O processo de trabalho se inicia com um acionamento realizado por um ente externo chamado acionador. Um processo de trabalho pode possuir vários tipos de acionamentos como entrada, onde cada tipo de acionamento pode ter um tipo de acionador correspondente. Como o processo de trabalho pode possuir mais de um tipo de acionamento, ele também pode possuir mais de um tipo de acionador. Ele pode ter, no máximo, um tipo de acionador para cada tipo de acionamento. Ele precisa ter, no mínimo, um tipo de acionamento para que possa ser iniciado. No entanto, pensando a respeito do número mínimo de tipos de acionadores, pode ocorrer a situação em que o processo de trabalho seja acionado pela passagem do tempo (um evento temporal). $\mathrm{O}$ que caracteriza a ausência de tipo de acionador, como é definido logo abaixo, fazendo com que a quantidade mínima de tipos de acionadores possa ser nula, apenas nesse caso.

Assim como o tipo de cliente, o tipo de acionador é um papel desempenhado por uma pessoa, um grupo de pessoas, um outro processo de trabalho, ou um dispositivo de TI, que é capaz de enviar um tipo de acionamento identificável e utilizável pelo processo de trabalho, como ilustrado na Figura 1.

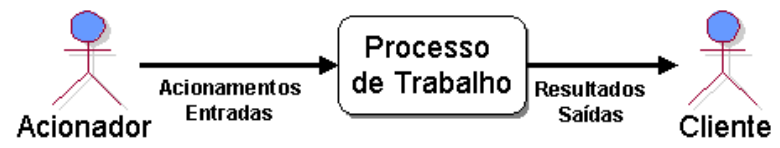

Figura 1. Processo de Trabalho

Um processo de trabalho está completamente identificado quando suas fronteiras, tanto de resultado quanto de acionamento, estão estabelecidas através da identificação de: todos os tipos de resultados, todos os tipos de clientes, todos os tipos de acionamentos e todos os tipos de acionadores. Caso pelo menos um destes elementos não tenha sido caracterizado, o processo de trabalho ainda não está completa e corretamente definido.

Esta conceituação de processo de trabalho permite que ele possa ser representado por um objeto, em conformidade com os preceitos da Orientação a Objeto, seguindo a idéia original de Jacobson (1994). Com efeito, um acionamento recebido pelo processo de trabalho pode ser visto como uma mensagem recebida, a qual aciona um comportamento deste objeto. De maneira similar, um resultado enviado para fora do processo de trabalho pode ser considerado como uma mensagem enviada para um outro objeto, o qual representa um cliente do processo de trabalho. Deste modo, tanto o processo de trabalho quanto o seu ambiente externo, formado por acionadores e clientes, podem ser representados por objetos. 
Retomando a conceituação geral de processo, como a de Davenport (1994) por exemplo, um processo é comumente reconhecido como um conjunto das atividades que devem ser executadas para atender um cliente. Portanto, o processo de trabalho também é considerado como sendo constituído por uma estrutura articulada de atividades, a qual pode ser representada por um grafo orientado, cujos nós são atividades e cujos arcos são acionamentos entre pares de atividades, conforme ilustrado na Figura 2.

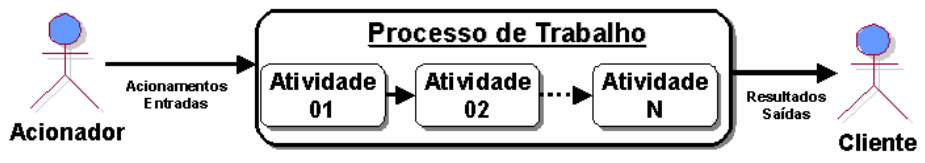

Figura 2. Grafo de atividades do Processo de Trabalho

Cada atividade identificada em um processo de trabalho deve ser caracterizada com os mesmos atributos estabelecidos para processo de trabalho, quais sejam: tipos de $\underline{\text { resultados}}$, tipos de clientes, tipos de acionamentos e tipos de acionadores. Isto significa que uma atividade pode ser tratada como um processo de trabalho, o que acontece com freqüência na prática, quando uma atividade pode ser detalhada como um processo, o qual neste caso é chamado de subprocesso.

Uma grande vantagem pode ser obtida quando o sistema computacional de informação usado para apoiar um processo de trabalho é estudado como sendo constituído pelo conjunto dos Casos de Uso existentes para apoiar algumas atividades do processo de trabalho. Nesta perspectiva, o papel exercido pelo executante da atividade é o próprio papel exercido pelo ator usuário do Caso de Uso correspondente.

O rigor utilizado para conceituar processo de trabalho e atividade, e a possível representação destes elementos através da Orientação a Objeto, conforme proposto anteriormente por Jacobson (1994), constituem uma base para a criação de um arcabouço conceitual que pode ser compartilhado por profissionais de TI e especialistas no negócio, ensejando o adequado entendimento do trabalho efetuado e a conseqüente análise de requisitos bem contextualizada e focada nos resultados para a organização.

\section{O Papel do Processo de Trabalho nas Organizações}

Uma organização pode ser vista como um conjunto de processos de trabalho, encadeados de modo a permitir que ela reaja corretamente quando ativada para produzir os resultados esperados por entidades externas. Este conjunto de processos de trabalho constitui uma estrutura modular, onde cada processo de trabalho representa um pedaço do trabalho total a ser realizado pela organização.

Para avaliar se uma estrutura de processos é lógica e eficiente, é necessário verificar se esses módulos têm "alta coesão" e "baixo acoplamento" (Page-Jones 1980). Para isso, é necessário que as fronteiras de cada processo do trabalho sejam minimizadas em quantidade de resultados e acionamentos. A situação ótima ocorre quando o processo do trabalho tem apenas um resultado e não mais do que um acionamento, tomando como base as definições da seção anterior.

Entretanto, não é fácil descrever um conjunto de processos de trabalho existentes nas organizações, porque elas geralmente são compostas por estruturas funcionais, ou seja, por setores especializados em executar certos tipos de atividades. Quando um processo do trabalho é limitado a um módulo funcional da organização (por exemplo 
uma seção, ou um departamento), a estrutura de processos tende a possuir "alto acoplamento" e "baixa coesão". Isto corresponde a uma modularização pobre e, conseqüentemente, a uma estrutura que se torna mais difícil de entender e operar.

Os autores que promoveram a abordagem de processos, tanto no controle de qualidade total (Campos 1992) como na reengenharia (Davenport 1994), enfatizaram que os processos importantes normalmente cruzam os limites da estrutura funcional da organização. Em geral, estes tipos de processos podem se beneficiar de sistemas de informação que podem lhes causar melhorias surpreendentes de desempenho.

O grande desafio inicial é a identificação dos processos de trabalho pertencentes ao escopo do problema-alvo. Neste assunto, as organizações freqüentemente possuem pouca documentação além da sua estrutura hierárquica funcional, e raramente têm alguma formalização sobre seus processos. Caso existam alguns processos definidos, eles provavelmente não seguem o rigor de nossa definição de processo de trabalho. $\mathrm{Na}$ nossa visão, esta é uma das principais razões para falhas de alinhamento das soluções de TI com os objetivos do negócio da organização.

\section{Conclusão e Trabalhos Futuros}

Neste artigo foi abordada a questão do alinhamento dos sistemas de informação com os objetivos das organizações, e foi introduzido o conceito de Processo de Trabalho para a obtenção de um entendimento mais formal sobre o trabalho realizado, a fim de permitir um compartilhamento de visões entre profissionais de TI e especialistas no negócio. As proposições apresentadas seguem a linha da MDA (Miller 2003) (Kleppe 2003), e da abordagem inovadora de Jacobson (1994), que procurou unir a objetividade da Reengenharia (Davenport 1994) com o rigor da Orientação a Objeto.

A utilização do conceito de processo de trabalho deve se disseminar pelas fases do ciclo de vida das aplicações de TI na organização. Isto pode proporcionar um bom rastreamento entre as especificações dos processos do negócio e as correspondentes especificações e detalhamentos dos aplicativos.

No entanto, para que esta idéia seja realizável, é necessário que as ferramentas (conceituais e computacionais) utilizadas na modelagem dos processos da organização sejam compatíveis ou até compartilhem o mesmo ambiente das ferramentas utilizadas na especificação e detalhamento dos softwares de aplicação. Em outras palavras, os modelos produzidos no nível CIM da MDA, que poderiam ser os modelos dos processos de trabalho, devem permitir o mapeamento de suas características para os modelos do nível PIM da MDA, que são em geral expressos na UML (Unified Modeling Language).

Uma amostra desta possibilidade, que foi mencionada no final na seção 4, é a associação de cada Caso de Uso a uma atividade de um processo de trabalho. Algumas experiências acadêmicas e práticas já realizadas pelos autores, indicam uma sensível melhoria no alinhamento com o negócio, de especificações de aplicações de TI que utilizem esse tipo de enfoque.

Um importante trabalho a ser ainda realizado, é o estabelecimento de um método completo para analisar e propor soluções de TI a partir do estudo dos processos de trabalho, estendendo os processos já conhecidos de Engenharia de Software para esta área um pouco anterior à análise de requisitos. Tal método deverá conter diretrizes e 
normas para a modelagem de processos de trabalho, e também deverá originar a criação de ferramentas que permitam o rastreamento dos requisitos, desde o seu nascedouro no processo de trabalho, até a especificação e o detalhamento das correspondentes características do sistema de informação computadorizado.

Este método deve ser desenvolvido de maneira iterativa, através da utilização de experimentações em situações reais que possam validá-lo efetivamente. As dificuldades são muito grandes para realizar este tipo de experiência nas organizações. Entretanto, já se tem notícia de algumas iniciativas nesta linha no Brasil, como, por exemplo, uma na COPEL (Companhia Paranaense de Energia) (Souza 2003).

O sucesso na determinação de um método como este, que complemente os esforços realizados na área de Processos de Qualidade de Software (Pressman 2001), poderá contribuir decisivamente para a Engenharia de Software tornar-se uma verdadeira área de engenharia, semelhante às outras mais tradicionais.

\section{Referências}

Campos, Vicente Falconi, (1992), "TQC: Controle da Qualidade Total (no estilo japonês)", Bloch Editora, Rio de Janeiro.

Davenport, Thomas H. (1994), "Reengenharia de Processos: Como inovar na empresa através da tecnologia da informação", Editora Campus, Rio de Janeiro.

Gulledge, Thomas (2001), "Aligning the Technology and Management Models: Business Process Management and Standard Software Solutions", Publicado em ITgestützte betriebswirtschaftliche Entscheidungsprozesse, Bernd Janke and Friederike Wall (Editors). Wiesbaden: Gabler Verlag, 2001.

Harmon, Paul (2003) "Business Process Change: A Manager's Guide to Improving, Redesigning, and Automating Processes", Morgan Kaufmann Publishers, San Francisco.

Jacobson, Ivar; Ericsson, Maria; Jacobson, Agneta (1994), "The Object Advantage: Business Process Reengineering with Object Technology", Addison-Wesley, USA.

Kleppe, Anneke G.; Warmer, Jos; Bast, Wim (2003), "MDA Explained: The Model Driven Architecture: Practice and Promise", Addisson Wesley, USA.

Miller, Joaquin; Mukerji, Jishnu (2003), "MDA Guide Version 1.0.1”, OMG - Object Management Group, Inc.", http://www.omg.org/cgi-bin/doc?omg/03-06-01, último acesso em junho/2005.

Page-Jones, Meilir (1980), "Practical Guide to Structured Systems Design”, Yourdon Press, New York, USA.

Pressman, Roger S. (2001) "Software Engineering: A Practitioner's Approach", 5. ed., McGraw-Hill, Boston.

Souza, Deise e Oliveira, Willian Lopes, (2003) , "Gestão por Processos na Área de TI", Anais do V Simpósio Internacional de Melhoria de Processo de Software, 3 a 5 de novembro de 2003, Recife, Brasil. 